\title{
Unmet needs in Italian cardiology: Acute cardiovascular syndrome in the new multi-ethnic society
}

\author{
Alice Sacco*, Nuccia Morici, Dario Brunelli, Giovanna Viola and Fabrizio Oliva \\ Intensive Cardiac Care Unit and De Gasperis Cardio Center, ASST Grande Ospedale Metropolitano Niguarda, Milan, Italy
}

\begin{abstract}
Background: In multi-ethnic societies it has frequently and repeatedly been shown that some minority groups have higher rates of traditional Coronary Artery Disease (CAD) risk factors, different rates of treatment with revascularization procedures, and excess morbidity and mortality from CAD when compared to the white population. In the last two decades Italy is becoming a diverse society with more than 5 million of inhabitants from minority ethnic groups: for this reason, we decided to investigate whether ethnic differences in our metropolitan area are similar compared with the experience of Western European countries.
\end{abstract}

Methods and results: We performed a retrospective cohort study of 359 patients with Acute Coronary Syndromes (ACS) hospitalized at Intensive Cardiac Care Unit (ICCU), Heart Center of ASST Grande Ospedale Metropolitano Niguarda, Milan, Italy between 2014 and the end of 2016. 7\% of these patients were South-Asian, Northern-African and Southern American. Despite the younger age of non-Italian patients, their comorbid conditions and traditional cardiovascular risk factors had similar rates. We did not observe any difference in terms of either intra-hospital or at follow-up mortality.

Conclusions: We believe that a better knowledge and understanding of epidemiological changes in Italian society could improve our clinical practice, in particular in order to better customize treatments and therapies.

\begin{abstract}
Abbreviations: CAD: Coronary Artery Disease; ACS: Acute Coronary Syndrome; ICCU: Intensive Cardiac Care Unit; GBD: Global Burden of Disease Study; IHD: Ischemic Heart Disease; WHO: World Health Organization; AMI: Acute Myocardial Infarction; PCI: Percutaneous Coronary Intervention.

\section{Introduction}

In the Western world, acute coronary syndrome (ACS) is the most common cause of death [1]. According to the Global Burden of Disease Study (GBD), Ischemic Heart Disease (IHD) is ranked first among the leading causes of mortality for eight regions in the world [2]. The World Health Organization (WHO) estimated that coronary artery disease (CAD) will be the single largest cause of disease burden in many countries world-wide by the year 2020 [3]. Similarly, in Italy, heart disease remains the leading cause of death [4]: overall, it accounts for about 1 in 5 deaths with CAD being responsible for over 75.000 deaths per year.
\end{abstract}

In the countries that have long tradition of existing multi-ethnic societies as Canada, US and UK it has frequently and repeatedly been shown that some minority groups have higher rates of traditional CAD risk factors, different rates of treatment with revascularization procedures, and excess morbidity and mortality from CAD when compared to the white population. Numerous investigations have been made into the causes of these disparities. Few studies from Canada, the United States, and Europe have highlighted the existence of ethnic differences in the incidence of Acute Myocardial Infarction (AMI) hospitalization, cardiac risk profile [5] and outcomes after AMI [6,7], Percutaneous Coronary Intervention (PCI), and coronary artery bypass graft [8].

The demographics of Italy are changing rapidly, and our country is becoming a diverse society with more than 5 million of the overall population from minority ethnic groups [9]: for this reason, we decided to highlight the increasing diversity of our patients affected by ACS.

\section{Methods}

Data were collected retrospectively in Milan, Italy between 2014 and 2016 and were analyzed according to patient ethnicity: we performed a retrospective cohort study of 359 patients with ACS hospitalized at the ICCU of ASST Grande Ospedale Metropolitano Niguarda. This is a tertiary care center with 1173 beds and over 90.000 visits per year at Emergency Department. Primary exposure was ethnicity and race, and outcomes were intrahospital and 6 months mortality rates.

Informed consent was obtained from each patient and the study protocol conforms to the ethical guidelines of the 1975 Declaration of Helsinki as reflected in a priori approval by the institution's human research committee.

Demographic, significant risk factors or past medical history, anthropometric, ACS stratum, treatment, length of hospitalization, outcome (alive, dead) and complications (such as bleeding) data were obtained from the medical records and subsequently entered into a centralized database with security password by trained coordinators. Standardized definitions for all patient-related variables and clinical

${ }^{*}$ Correspondence to: Alice Sacco, Dipartimento Cardio-toraco-vascolare, Intensive Coronary Care Unit and De Gasperis Cardio Center, ASST Grande Ospedale Metropolitano Niguarda, Piazza Ospedale Maggiore, 3 Milan 20162, Italy, Tel: +390264442565, E-mail: alice.sacco@ospedaleniguarda.it

Key words: cardiogenic shock; acute heart failure

Received: November 06, 2018 ; Accepted: November 30, 2018; Published: December 10, 2018 
diagnoses were used [10,11]. Precise definition and quantification for risk factors, past medical history and ways of treatments were described in earlier publications $[10,11]$.

We compared demographics, clinical and angiographic characteristics of recruited patients using patient ethnicity. The ShapiroWilk test of normality was performed, and continuous variables showed a skewed distribution. Therefore, they were compared using the MannWhitney test by ranks and the results were presented as median and 25th and 75th percentile. Discrete variables were compared using the chi squared test and presented as absolute and relative frequencies per category. Analyses were performed using a commercially available statistical package STATA/SE 14 (StataCorp LP, College Station, TX).

\section{Results}

There were 359 patients affected by ACS from 2014 to 2016 hospitalized at our ICCU: of these 333 (93\%) were Italian; 26 (7\%) were South-Asian, Northern-African and Southern American patients. Ethnic subgroups are shown in Figure 1 and patient characteristics are listed in Table 1. The distribution was as follows: $44.0 \%$ Northern Africans, 36\% Southern Asiatics and 20\% Southern Americans. Around $71 \%$ of patients, Italians and not, were males. The mean age of Italian patients at presentation was $71(62-81)$ years versus 57 (48-65) years with $p<0.001$. Despite the younger age of non-Italian patients, their comorbid conditions and traditional cardiovascular risk factors had similar rates: a large proportion of individuals within the two groups and also within each ethnic group had more than two coronary risk factors including hypertension, diabetes mellitus and dyslipidaemia. In both groups there was a high rate of current smokers (26.1 vs $28.0 p=$ 0.830 ). The rate of occurrence of a previous AMI was similar (30.2 vs $24.0 p=0.511)$. GRACE score was similar [151 (80-227) vs 163 (66-251) with $p=0.715$ ], whereas CRUSADE score was higher in non-Italians [31 (17-52) vs 18 (10-36) with $p<0.034]$. We did not observe differences in recommended therapies at discharge: in particular as far as it concerns

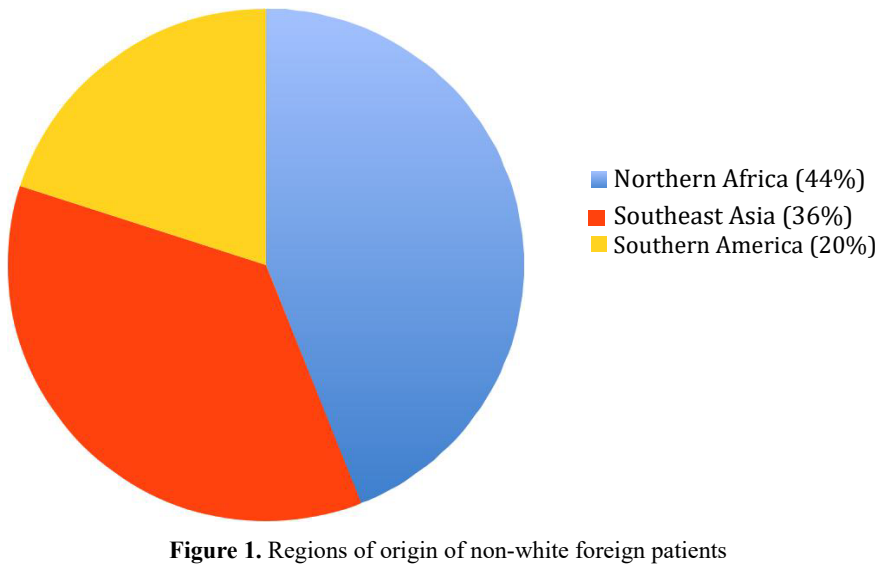

Table 1. Patient characteristics

\begin{tabular}{|c|c|c|c|c|}
\hline & $\begin{array}{c}\text { Over all } \\
(359)\end{array}$ & $\begin{array}{l}\text { White Italians } \\
\text { (333 pts) }\end{array}$ & $\begin{array}{l}\text { Others } \\
\text { (26 pts) }\end{array}$ & $p$ value \\
\hline Age & $70(61-81)$ & $71(62-81)$ & $57(48-65)$ & $<0.001$ \\
\hline Males (\%) & $255(71.0)$ & $238(71.3)$ & $17(68.0)$ & 0.729 \\
\hline Diabetes mellitus (\%) & $113(31.5)$ & $106(31.7)$ & $7(28.0)$ & 0.698 \\
\hline Arterial hypertension (\%) & $232(64.6)$ & $221(66.2)$ & $11(44.0)$ & 0.025 \\
\hline Dyslipidemia (\%) & $185(51.5)$ & $175(52.4)$ & $10(40.0)$ & 0.232 \\
\hline Smoking (\%) & $94(26.2)$ & $87(26.1)$ & $7(28.0)$ & 0.830 \\
\hline Previous myocardial infarction (\%) & $107(29.8)$ & $101(30.2)$ & $6(24.0)$ & 0.511 \\
\hline Ejection fraction (\%) & $50(40-55)$ & $50(40-55)$ & $50(40-55)$ & 0854 \\
\hline Haemoglobin $(\mathrm{g} / \mathrm{dL})$ at presentation & $13.5(11.9-14.9)$ & $13.5(11.9-14.9)$ & $13.5(11.9-14.9)$ & 0.393 \\
\hline Platelets at presentation, 109/L & $220(178-263)$ & $220(178-263)$ & $215(182-242)$ & 0.726 \\
\hline Creatinine at presentation, $\mathrm{mg} / \mathrm{dl}$ & $0.92(0.77-1.13)$ & $0.92(0.77-1.13)$ & $0.92(0.77-1.06)$ & 0.478 \\
\hline STEMI & $173(48.2)$ & $162(48.5)$ & $11(44.0)$ & 0.871 \\
\hline GRACE score & $152(78-230)$ & $151(80-227)$ & $163(66-251)$ & 0.715 \\
\hline CRUSADE & $30(15-52)$ & $31(17-52)$ & $18(10-36)$ & 0.034 \\
\hline PCI & $282(78.6)$ & $265(79.3)$ & $17(68.0)$ & 0.378 \\
\hline Ticagrelor at discharge & $96(26.7)$ & $90(26.9)$ & $6(24.0)$ & 0.912 \\
\hline Prasugrel at discharge & $89(24.8)$ & $82(24.5)$ & $7(28.0)$ & 0.897 \\
\hline Statin at discharge & $306(85.2)$ & $287(85.9)$ & $19(76.0)$ & 0.364 \\
\hline Betablocker at discharge & $294(81.9)$ & $273(81.7)$ & $21(84.0)$ & 0.933 \\
\hline Ace inhibitor at discharge & $225(62.7)$ & $208(62.3)$ & $17(68.0)$ & 0.826 \\
\hline In-hospital death & $17(4.74)$ & $15(4.5)$ & $2(8.0)$ & 0.426 \\
\hline Death at follow up & $35(9.75)$ & $33(9.9)$ & $2(8.0)$ & 0.760 \\
\hline Re-infarction at follow-up & $25(6.96)$ & $24(7.2)$ & $1(4.0)$ & 0.531 \\
\hline Stroke at follow-up & $4(1.1)$ & $4(1.2)$ & 0 & 0.528 \\
\hline Follow-up (months) & $15(11.22)$ & $15(11.22)$ & $13(4.20)$ & 0.135 \\
\hline
\end{tabular}


double antiplatelets therapy, ACE-inhibitors, beta-blockers and statins. We did not observe any difference in terms of either intra-hospital or at follow-up mortality.

\section{Discussion}

As far as we know this is the first Italian attempt to examine ethnic differences in risk of $\mathrm{CAD}$ in a small contemporary population affected by ACS.

The rate of occurrence of a previous AMI was similar in white Italian and non-white patients, even though foreigners were significantly younger: this could be explained by the same rate of comorbidities and cardiovascular risk factors, again despite the younger age of non-white patients.

We can probably explain the higher CRUSADE score in nonItalians patients because of a slightly higher systemic arterial pressure at presentation.

There were not differences in recommended therapies at discharge and we did not observe a trend towards a higher mortality, either intrahospital or at follow-up.

The similar mortality trend in foreign and Italians patients included in our registry, even though the younger age of foreigners, are incompletely understood: the role of classical cardiovascular risk factors is clearly important despite the patterns of these risk factors varying by ethnic group; socioeconomic factors are most probably underestimated even though there was equal access to care between Italian and nonItalian patients.

The main study limitation is the small cohort: this is the reason we could not generalize estimating equations to test for associations between ethnicity and race and outcomes, but still we could infer some interesting observations.

We are aware that this small cohort of patients represents a wide ethnic heterogeneity, encompassing wide variety of genotypes, cultures, diets, belief systems, educational attainment, socioeconomic status and risk factors. To cite just one significant difference apart from traditional cardiovascular risk factors, previous studies reported different presentations of ACS between British South Asians, as a whole, and white Europeans [12].

It is well known that ethnic subgroups are underrepresented in large randomized clinical trials: Plato trial [13], for example, included only 34 black subjects. A recent pilot cross-over study14 with these 34 black patients examined the onset and degree of platelet inhibition in these subjects with stable coronary disease taking ticagrelor compared to those already taking clopidogrel. Ticagrelor provided greater platelet inhibition than clopidogrel.

Some of the ethnic subgroups residents in Italy have been underrepresented in research and as a consequence a multitude of guidelines exists for the general population; moreover, specific reference and recommendation on primary and secondary prevention guidelines in relation to ethnic groups is extremely limited.

There remain significant challenges to understanding racial/ethnic differences in cardiovascular risk factors and to including minority groups in clinical trials of therapeutic interventions, thereby ensuring optimal treatment for all.

Our data are a further demonstration that ethnic variations in the incidence and, possibly, clinical outcome of acute CAD events exist.
The causes for such differences, however, are multiple and difficult to fully address, given the complex interplay of contributing factors. In the meantime, fighting against classical risk factors, which have consistently been associated with CAD events, and intervening timely and appropriately in patients with a suspicion of an ACS remain the major goals for decreasing CAD incidence and mortality in any kind of ethnic groups. In fact the similar incidence of cardiovascular death of the two groups could mean that if we involve the foreign patients in a well-established follow-up program we can improve the outcomes at long term: the younger age at presentation of the non Italian ethnic groups highlights that we need a more focused research in the field of "multi-ethnic cardiology", along with public health efforts focused on reducing health risks and their related comorbidities in these groups of patients. In particular the care for ACS and MI could further improve in quality, benefiting all racial and ethnic groups, thanks to large national quality control registries.

In conclusion we believe that a better knowledge and understanding of epidemiological changes in Italian society could improve our clinical practice: awareness of ethnic heterogeneity in IHD could help to better customize treatments and therapies and therefore to improve secondary prevention and outcomes in these subgroups of patients.

\section{Conflicts of interest}

Alice Sacco has received consulting fees and honoraria from Orion, outside the submitted work; Nuccia Morici has received consulting fees and honoraria from AstraZeneca, Daiichi-Sankyo, Eli Lilly, and Orion, outside the submitted work; Dario Brunelli and Giovanna Viola have no conflicts of interest to declare; Fabrizio Oliva has received consulting fees and honoraria from Medtronic, Orion, Servier and AstraZeneca, outside the submitted work.

\section{References}

1. Herzog E, Saint-Jacques H, Rozanki A (2004) The pain pathway as a tool to bridge the gap between evidence and management of acute coronary syndrome. Crit Pathw Cardiol 3: 20-24 [Crossref]

2. Murray CJ, Lopez AD (1997) Mortality by cause for eight regions of the world: Global Burden of Disease Study. Lancet 349: 1269-1276. [Crossref]

3. WHO. The World Health Statistic (2012). Available from: http://www.who.int/gho/ publications/world_health_statistics/EN_WHS2012_Full.pdf

4. AIRTUM Working Group, CCM, AIEOP Working Group (2013) Italian cancer figures, report 2012: Cancer in children and adolescents. Epidemiol Prev 37: 1-225. [Crossref]

5. Nijjar AP, Wang H, Quan H, Khan NA (2010) Ethnic and sex differences in the incidence of hospitalized acute myocardial infarction: British Columbia, Canada 1995 2002. BMC Cardiovasc Disord 10: 38 [Crossref]

6. Khan NA, Grubisic M, Hemmelgarn B, Humphries K, King KM, et al. (2010) Outcomes after acute myocardial infarction in South Asian, Chinese, and white patients. Circulation 122:1570-1577. [Crossref]

7. Gupta M, Doobay AV, Singh N, Anand SS, Raja F, et al. (2002) Risk factors, hospital management and outcomes after acute myocardial infarction in South Asian Canadians and matched control subjects. CMAJ 166: 717-722 [Crossref]

8. Gasevic D, Khan NA, Qian H, Karim S, Simkus G, et al. (2013) Outcomes following percutaneous coronary intervention and coronary artery bypass grafting surgery in Chinese, South Asian and White patients with acute myocardial infarction: administrative data analysis. BMC Cardiovasc Disord 13:121 [Crossref]

9. Cittadini Stranieri. Popolazione residente e bilancio demografico al 31 dicembre 2015 , in ISTAT, 23 September 2016

10. Task Force on the management of ST-segment elevation acute myocardial infarction of the European Society of Cardiology (ESC), Steg PG, James SK, Atar D, Badano LP, et al. (2012) ESC Guidelines for the management of acute myocardial infarction in patients presenting with ST-segment elevation. Eur Heart $J$ 33: 2569-2619. [Crossref] 
11. Roffi M, Patrono C, Collet JP, Mueller C, Valgimigli M, et al. (2016) 2015 ESC Guidelines for the management of acute coronary syndromes in patients presenting without persistent ST-segment elevation: Task Force for the Management of Acute Coronary Syndromes in Patients Presenting without Persistent ST-Segment Elevation of the European Society of Cardiology (ESC). Eur Heart J 37: 267-315 [Crossref]

12. Teoh M, Lalondrelle S, Roughton M, Grocott-Mason R, Dubrey SW (2007) Acute coronary syndromes and their presentation in Asians and Caucasian patients in Britain. Heart 93: 183-188. [Crossref]
13. Wallentin L, Becker RC, Budaj A, Cannon CP, Emanuelsson H, et al. (2009)Ticagrelor versus Clopidogrel in Patients with Acute Coronary Syndromes $N$ Engl J Med 361 : 1045-1057 [Crossref]

14. Waksman R, Maya J, Angiolillo DJ, Carlson GF, Teng R, et al. (2015) Ticagrelor versus clopidogrel in black patients with stable coronary artery disease prospective, randomized, open-label, multiple-dose, crossover pilot study. Circ Cardiovasc Intery 8: e002232. [Crossref]

Copyright: (C2018 Sacco A. This is an open-access article distributed under the terms of the Creative Commons Attribution License, which permits unrestricted use, distribution, and reproduction in any medium, provided the original author and source are credited. 